\title{
Vibrational fingerprint of the absorption properties of UiO-type MOF materials
}

\author{
Andy Van Yperen-De Deyne ${ }^{1,4} \cdot$ Kevin Hendrickx ${ }^{1,2} \cdot$ Louis Vanduyfhuys ${ }^{1}$. \\ German Sastre $^{3}$ Pascal Van Der Voort ${ }^{2}$. Veronique Van Speybroeck ${ }^{1}$. \\ Karen Hemelsoet ${ }^{1}$
}

Received: 14 September 2015 / Accepted: 17 February 2016

(C) Springer-Verlag Berlin Heidelberg 2016

\begin{abstract}
The absorption properties of UiO-type metalorganic frameworks are computed using TD-DFT simulations on the organic linkers. A set of nine isoreticular structures, including the UiO-66 and UiO-67 materials and functionalized variants, are examined. The excitation energies from a static geometry optimization are compared with dynamic averages obtained from sampling the ground-state potential energy surface using molecular dynamics. The vibrational modes that impact the excitation energy are identified. This analysis is done using a recently proposed tool based on power spectra of the velocities and the excitation energies. The applied procedure allows including important factors influencing the absorption spectra, such as the periodic framework, linker variation and dynamical effects including harmonic and anharmonic nuclear
\end{abstract}

Published as part of the special collection of articles "Health \& Energy from the Sun".

Electronic supplementary material The online version of this article (doi:10.1007/s00214-016-1842-8) contains supplementary material, which is available to authorized users.

Karen Hemelsoet

karen.hemelsoet@ugent.be

1 Center for Molecular Modeling (CMM), Ghent University, Technologiepark 903, 9052 Zwijnaarde, Belgium

2 Department of Inorganic and Physical Chemistry, Center for Ordered Materials, Organometallics and Catalysis (COMOC), Ghent University, Krijgslaan 281 (S3), 9000 Ghent, Belgium

3 Instituto de Tecnologia Quimica UPV-CSIC, Universidad Politecnica de Valencia, Av. Los Naranjos s/n, 46022 Valencia, Spain

4 Present Address: ArcelorMittal Gent, John Kennedylaan 51, 9042 Ghent, Belgium motions. This methodology allows investigating in detail the vibrational fingerprint of the excitation energy of advanced materials such as MOFs and gives perspectives to tailor materials toward new light-based applications.

Keywords Power spectra $\cdot$ Molecular dynamics $\cdot$ UiO frameworks $\cdot$ TD-DFT $\cdot$ Vibrational modes

\section{Introduction}

Metal-organic frameworks (MOFs) define a versatile class of crystalline, porous materials, constructed from inorganic clusters that are linked by organic moieties [1-5]. Owing to the large number of both organic and inorganic building blocks available, MOFs have a high level of tunability. In combination with the large pores and high surface area, this explains why these materials are considered for many potential applications, in particular in the fields of catalysis, gas storage, chemical sensing and separation [6-8]. More recently, the research domain of MOFs is extended toward light-based applications, more specifically their use in photocatalysis $[9,10]$ and as luminescent materials [11].

In this view, the class of UiO-66 materials has been thoroughly investigated, giving special attention to how the tunability of the framework affects the light-related properties [12]. UiO-66 frameworks consist of Zr-O octahedron corners and benzodicarboxylic acid linkers (BDC). The structure of the framework and the different building units are shown in Fig. 1. These materials are known to be very stable and have been intensively studied for a whole range of applications [13-18]. A synergic combination of experimental XRPD and EXAFS measurements and theoretical $a b$ initio simulations proved necessary to reveal the correct geometrical structure of UiO-66, as reported by Valenzano 
Fig. 1 Structure of the UiO-66 porous framework: a UiO-66 shown along the $a$-axis, the primitive unit cell is explicitly indicated. b Building units: an inorganic $\mathrm{Zr}_{6} \mathrm{O}_{4}(\mathrm{OH})_{4}$ unit and an organic BDC linker. Atoms are colored as follows:

C: gray/black; O: red; H: white; Zr: light blue

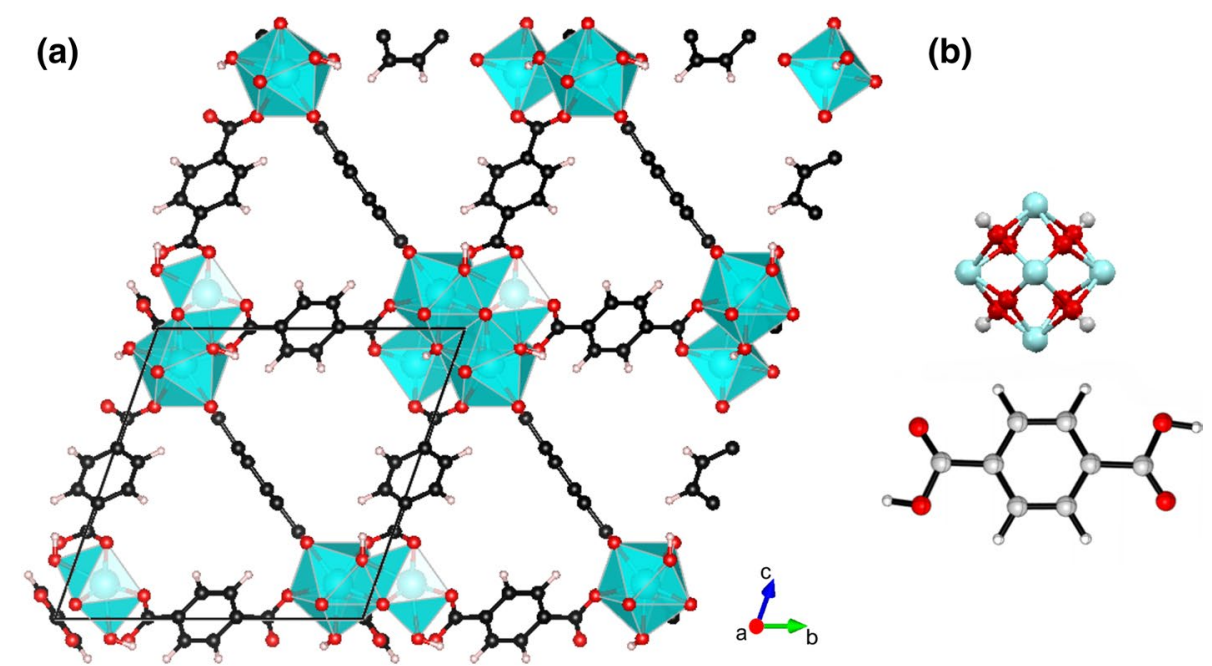

et al. [19]. In addition to the prototype UiO-66 material, the amino-functionalized variants have been studied for photocatalytic applications. Garcia et al. showed that the $\mathrm{NH}_{2}$ group shifts the absorption spectrum toward the visible range [20]. Sun et al. [21] proposed a ligand-to-metal charge transfer (LMCT) occurring in the framework, based on photoluminescence data. Theoretically, however, this LMCT transfer in the visible range seems less efficient than expected [23]. Other ligand substituents have also been considered, using experimental or theoretical methods or a combination thereof. Shen et al. could correlate the rate coefficients with the Hammett coefficients and proposed that the functional groups would promote charge transport and separation of the carriers in the framework [23].

Recent studies on the periodic UiO materials confirmed the experimental band gap modulations via calculations based on density functional theory (DFT) and attributed it to an extra electronic band appearing in the materials band gap [24, 25]. Some of the presenting authors also investigated the optical characteristics of mono- and disubstituted UiO-66-X frameworks (with $\mathrm{X}=\mathrm{OH}, \mathrm{NH}_{2}$ and $\mathrm{SH})$ using ab initio methods in direct comparison with experimental UV/Vis measurements [23]. The theoretical spectra were obtained using time-dependent DFT (TDDFT). To enhance the link between the experimental and theoretical conditions, temperature effects were included via molecular dynamics (MD) simulations on the isolated linkers allowing to include their inherent flexibility. Subsequently, averaged absorption properties were obtained using snapshots generated during the ab initio MD runs. Distinct influences are found from the nuclear dynamics on the calculated excitation energies. However, at that point, the precise origin of these differences was unclear and this inspired us to perform a more in-depth analysis. This study followed the procedure as proposed by the group of Barone et al., who demonstrated that averaging over different configurations-generated during an MD simulationleads to reliable dynamic estimations of transition properties in good agreement with experimental data [26-29]. Such methodology was previously successfully applied for a large variety of systems, ranging from small gas phase molecules over solvated dyes to dyes occluded in a porous zeolitic environment [29-32]. In the majority of the studies, the electronic transitions are calculated using TD-DFT in its linear response formulation, since it offers a good accuracy/computational cost ratio [33-35].

The aim of the present contribution is to gain a better understanding of which vibrational modes affect calculated electronic transitions in UiO-type materials. To this end, we will compare static excitation energies with dynamic averages, obtained using distorted geometries generated during MD runs. This is done for 9 UiO-type frameworks, bearing a variety of organic linkers as shown in Fig. 2, to study induced effects on the excitation spectra. The top figure represents BDC and BPDC (4,4'-biphenyl-dicarboxylate) linkers present in the pristine UiO-66 and UiO-67 materials and some other functionalized derivatives. These structures have previously been synthesized by various groups, and their absorption properties and/or photocatalytic performance have been tested [19-23]. Additionally, Fig. 2 includes 2 aliphatic linker models, constituting UiO-66-oxal and UiO-66-CC. These hypothetical UiOs are included as they can provide more insight in the framework motions due to their reduced set of possible vibrations. Due to their reduced conjugation compared to the aromatic linkers, these materials are not expected to absorb in the visual range. All frameworks share the same octahedral $\mathrm{Zr}_{6} \mathrm{O}_{4}(\mathrm{OH})_{4}$ inorganic building unit. Herein we want to explore which vibrational modes impact the observed absorption spectrum. We will employ an analysis method recently proposed by some of the presenting authors, which is based on a comparison of the velocity power spectra 
<smiles>[X]c1cc(C(=O)O)ccc1C(=O)O</smiles><smiles>[X]c1cc(C(=O)O)c([X])cc1C(=O)O</smiles><smiles>[X]c1cc(C(=O)O)ccc1-c1ccc(C(=O)O)cc1[X]</smiles>

UiO-66-X $\mathrm{X}=\mathrm{H}, \mathrm{OH}, \mathrm{NH}_{2}$
UiO-66-2,5X $\mathrm{X}=\mathrm{OH}, \mathrm{NH}_{2}$
UiO-67-2,5'X $\mathrm{X}=\mathrm{H}, \mathrm{NH}_{2}$<smiles>O=C(O)C(=O)O</smiles>

UiO-66-oxal

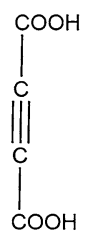

UiO-66-CC
Fig. 2 Representation of linkers studied in this work. When $\mathrm{X}=\mathrm{H}$, the materials are called UiO-66 and UiO-67

(VPS) with the power spectra of the transition energies (the so-called $\epsilon \mathrm{PS}$ ) [36]. By comparison with both power spectra, the vibrational fingerprint of the excitation energies can be unraveled. Here, the influence of the UiO framework will be explicitly included via MD simulations on the fully periodic structure. By comparing vibrational spectra of the pure linker versus the fully periodic UiOs, the influence of the UiO framework on the characteristic vibrational frequencies and absorption properties can be directly assessed. This is the first time the procedure based on the comparison of power spectra is used on large extended systems such as the UiO materials. The complete understanding of the light absorption properties is important for the further development of these frameworks as photoactive materials.

Overall, elucidating the vibrational features of complex materials offers detailed molecular-scale information. It is well known that IR spectroscopy can be of great help in the characterization of MOF materials [37]. In addition to numerous experimental studies, theoretical examinations are ideally suited to provide very detailed information on the geometrical structure of the constituent atoms and building units of the porous materials. Thus far, vibrational frequencies based on static quantum-mechanical calculations within the harmonic oscillator approximation are usually employed [38]. The calculated frequencies can be scaled to reproduce well-known characteristic peaks, in order to account for lack of anharmonicity and errors due to the employed level of theory. For the UiO-66- and UiO-67type materials, IR spectra were obtained using such a static ab initio approach [19, 39]. Valenzano et al. found a good agreement between harmonic frequencies of the hydroxylated and dehydroxylated UiO-66 framework and experimental FTIR data. The theoretical periodic data allowed to assign the main IR peaks, and moreover, changes in the skeletal modes (between 400 and $800 \mathrm{~cm}^{-1}$ ) suggested clear distortions of the inorganic $\mathrm{ZrO}$ units upon (de)hydration [19]. From the same group, a similar study on the UiO-67 material appeared, assigning in detail the observed IR peaks [39]. More recently, VPS derived from MD simulations have become an interesting alternative to static frequencies, including the anharmonicity of the surface as well as temperature effects in a natural way. To date, VPS of zeolitic materials have been calculated successfully to elucidate the framework vibrations [40], but this is not yet a routine strategy for MOFs. Leus et al. [41] reported VPS of Au nanoparticles loaded in UiO-66, which were found to be in good agreement with experimental spectra. Furthermore, Greathouse and Allendorf [42] obtained VPS from force field data in order to discuss the slow torsional motion $\left(40 \mathrm{~cm}^{-1}\right)$ of the aromatic rings in IRMOF-1 which they validated via NMR measurements. The provided vibrational fingerprint of the set of isoreticular UiOs will contribute to previous investigations. A detailed analysis of the VPS and the link with the excitation properties is thus far missing.

This paper is structured as follows. In Sect. 2, a brief summary is given on the calculation and interpretation of power spectra using either velocities or a spectroscopic property. Section 3 contains the computational details. The optical properties and vibrational fingerprints of the UiO frameworks are discussed in Sect. 4, starting with an overview of the calculated absorption data (Sect. 4.1), followed by an examination of the characteristic frequencies (Sect. 4.2) and, finally, in-depth discussion of the link between the vibrational motions and changes in the excitation energies (Sect. 4.3). We end by formulating the conclusions in Sect. 5.

\section{Methodology to calculate VPS and $\epsilon$ PS}

A velocity power spectrum (VPS) generated by a MD simulation contains all information about the internal vibrational modes (associated with vibrational coordinates $Q_{i}$ ) with frequencies $\omega_{i}$ and hence expresses the change in the molecular structure during the MD run [43, 44]. The VPS is defined as [44]:

$I_{\mathrm{VPS}}(\omega)=\sum_{\alpha=x, y, z} \sum_{l=1}^{N}\left|\int v_{l, \alpha}(t) \exp (-i \omega t) \mathrm{d} t\right|^{2}$, 
for a system with $N$ nuclei and where $v_{l, \alpha}$ is the $\alpha$ th Cartesian component of the velocity of the $l^{\prime}$ th nucleus. The VPS show high intensities at frequencies corresponding to the vibrational energy levels. Selecting particular atoms and hence constructing partial VPS allow to further refine the location of each mode. The decomposition of VPS into molecular motions is not straightforward for large molecular systems. A principal component analysis (PCA) [45] will be performed to determine the coordinates $Q_{i}$. PCA is in particular suitable for describing global motions of the system under investigation. Further details of the analysis are given in Ref. [36]. Sampling of the ground-state potential energy surface and subsequent computation of the vertical excitation energy $\epsilon$ for distinct snapshots creates a time series of $\epsilon(n)$ values. The excitation energy $\epsilon$ hence becomes a function of time and similar to the VPS, and the corresponding power spectrum of the excitation energy $(\epsilon$ PS) is characteristic of fluctuations in $\epsilon$. It is defined as

$I_{\epsilon P S}(\omega)=\left|\int \epsilon(t) \exp (-i \omega t) \mathrm{d} t\right|^{2}$.

Fig. 3 Schematic representation of the computational analysis tool based on VPS and $\epsilon$ PS. a Comparison of VPS with $\epsilon$ PS and $\epsilon \mathrm{PS}$ with a scaling of the frequency axis of $1 / 2$ allows to identify linearly or quadratically active modes. b The linearly active coordinate does not lead to a shift between the dynamic average $\langle\epsilon\rangle_{\text {dyn }}$ and static $\epsilon_{\text {stat }}$ excitation energy. c The quadratically active coordinate leads to a shift between the dynamic average $\langle\epsilon\rangle_{\text {dyn }}$ and static $\epsilon_{\text {stat }}$ excitation energy
In Ref. [36] it was reported that one needs to examine the overlap between $I_{\epsilon P S}$ and $I_{\mathrm{VPS}}$ to determine the correlations between internal motions and the electronic spectrum generated by the vertical transition energy $\epsilon$. Figure 3 conceptually clarifies this computational analysis. Peaks coinciding in the $\epsilon$ PS and VPS correspond to coordinates for which the excitation energy changes linearly, indicated as linearly active coordinates in Fig. 3. This can however not explain a shift of the average excitation energy obtained via the dynamic approach (defined as $\langle\epsilon\rangle_{\text {dyn }}$ ) to the static excitation (defined as $\epsilon_{\text {stat }}$ )-since they coincide-if this coordinate is sufficiently sampled. This shift is caused by higher order, in particular quadratic, terms. The quadratic contributions can be identified by comparing the $\epsilon \mathrm{PS}$ where the frequency axis is now scaled with $1 / 2$ with the original VPS. In case of an overlap in this situation, the corresponding vibrational mode of the quadratically active coordinate contributes to the shift between the static and dynamic excitation energy (see Fig. 3). For further details, we refer to our previous investigation [36].
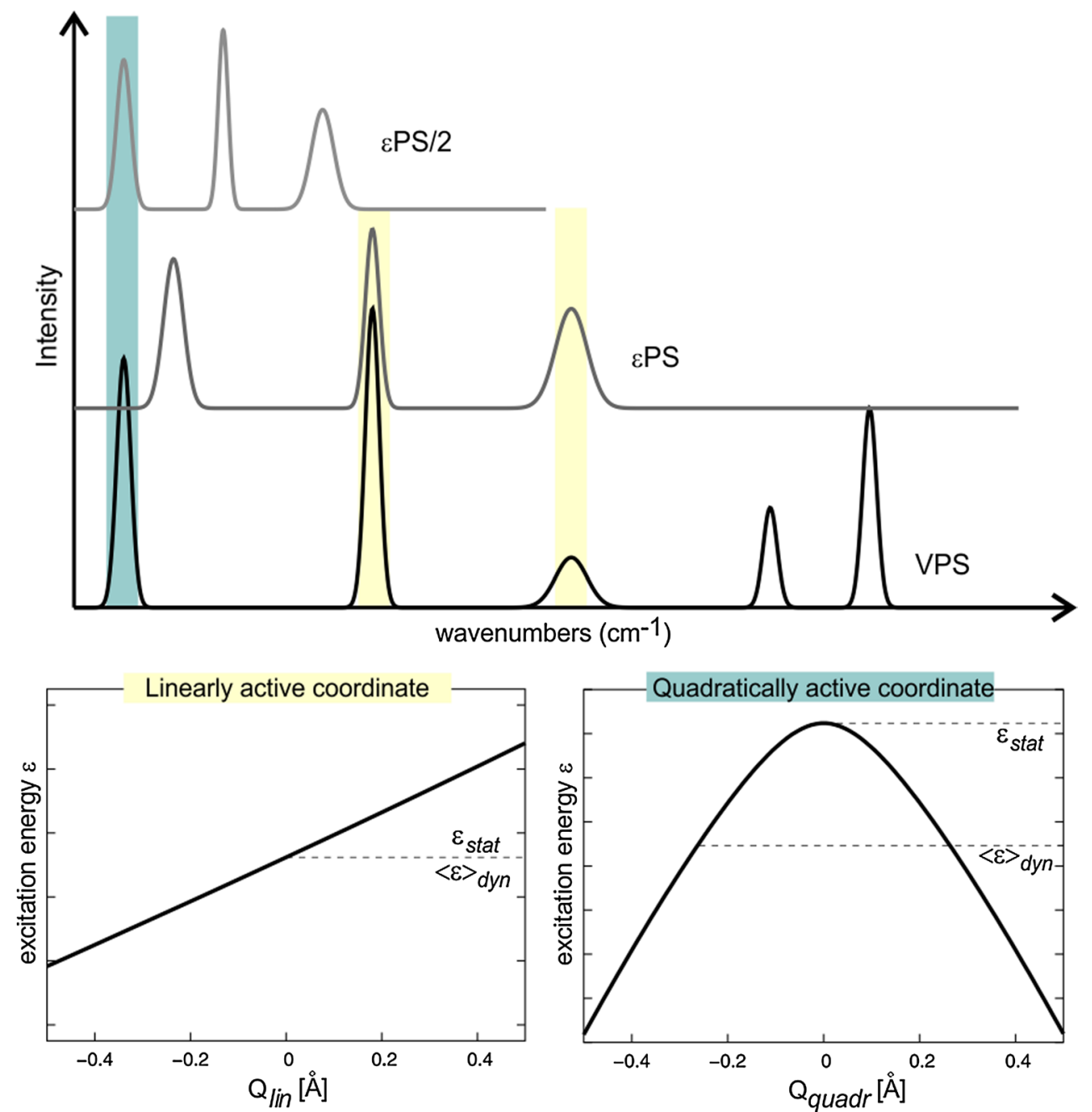


\section{Computational details}

Geometries are generated using a static geometry optimization or via dynamics simulations. The potential energy surface of the ground state is sampled using molecular dynamics on a DFT level of theory (Sect. 3.1). This sampling allows to include temperature as well as anharmonic effects. The sampling involves either the finite organic linker in gas phase (referred to as 'gas phase') or the fully periodic UiO (referred to as 'periodic'). Thereafter, a large number of snapshots are extracted from the generated MD runs, and for each snapshot, a static TD-DFT simulation is performed on a finite model representing the organic linker (Sect. 3.2). These resulting excitation energies will be systematically labeled 'dynamic', in contrast to the values involving the optimized structures which will be labeled 'static'.

\subsection{DFT simulations for the generation of geometries}

$\mathrm{Ab}$ initio optimizations and molecular dynamics (AIMD) simulations on both the isolated linkers and full periodic UiO-type frameworks were carried out using the CP2K software package [46]. They were performed using the PBE functional. Empirical dispersion corrections (DFTD3) were added to include long-range van der Waals interactions [47]. These ab initio simulations were done with the Gaussian and plane waves (GPW) method [48], using a DZVP Gaussian basis set and a plane wave basis set with a cutoff of 350 Ry using the Goedecker-Teter-Hutter pseudopotentials [49, 50].

For the AIMD simulations, the systems were initially heated to the desired temperature of $300 \mathrm{~K}$ using a CSVR thermostat [51], followed by MD runs using a Nosé-Hoover thermostat consisting of 5 coupled heat baths $[52,53]$. The total sampling time of the NVT simulations at $300 \mathrm{~K}$ was 35 ps for the gas phase linkers and 27 ps for the full $\mathrm{UiO}$ frameworks using a 0.5 -fs time step. Fully equilibrated trajectories of 20 ps were found sufficient for the computation of resolved power spectra. Subsequently, snapshots were taken every 5 fs (resulting in 4000 snapshots) and used as input for a static TD-DFT calculation to derive dynamic averages of the excitation energy (see hereafter Sect. 3.2). This methodology and similar level of theory (employing BLYP instead of PBE) have proven to yield accurate results for these types of systems $[22,31]$. The PBE functional is used in this work in correspondence with Refs. [14, 54, 55].

For the gas phase linkers, a cubic box [cell length 17.0 $\AA(\mathrm{UiO}-66)$ or $22.0 \AA(\mathrm{UiO}-67)]$ was employed, which is sufficiently large to avoid interactions between the molecules. The MOF frameworks were simulated starting from a trigonal unit cell. The AIMD simulations on the entire
UiOs were preceded by a cell and geometry optimization, performed at the level of theory described above. In the case of the original UiO-66 framework, this cell contains 114 atoms, i.e., 6 linkers and one $\mathrm{Zr}$-O building block (see Fig. 1). The optimized cell parameters are given in Table $\mathrm{S} 1$ of the Supplementary Material. The periodic frameworks of the UiO-66-oxal and UiO-66-CC structures are shown in Fig. S2 of the Supplementary Material.

\subsection{TD-DFT calculations on linker models}

Static calculations of the vertical excitation energy on the linkers-either obtained from a static geometry optimization or extracted from the MD runs-were done in Gaussian09 (G09) [56] using TD-DFT. In case of the AIMD runs on the periodic UiOs, the extracted linkers were terminated with two additional $\mathrm{H}$ atoms according to the procedure described in the Supplementary Material (section S2). The hybrid B3LYP [57, 58] functional in combination with a Pople 6-311+G(d,p) basis set [59] was employed, in line with previous studies reporting that this method provides reliable absorption data of organic structures of similar size $[23,60]$. Solvent effects ( $N, N$-Dimethylformamide, DMF) were included during TD-DFT calculations using a bulk solvent model, in particular a polarizable continuum model ( $\varepsilon=37.219)$ within the integral equation formalism [61], as is implemented in the G09 package. Vertical transitions $\epsilon$ can be considered as an initial approximation for experimental UV/Vis spectra and can provide clear insight into relative trends when investigating the influence of linker substitution [22, 31]. Absorption intensities are expressed in units of molar absorptivity $(\mathrm{L} / \mathrm{mol} \mathrm{cm})$ [see the Supplementary Material (section S3)].

\section{Results and discussion}

\subsection{Dynamic and static excitation energies}

We start the analysis of the light absorption properties of the UiO-type materials under investigation by examining the calculated excitation energies of the organic units. Table 1 lists the first excitation energies of the linkers, calculated using a static calculation ('static') or via the averaging procedure as explained in the computational details ('dynamic'). The dynamic averages result from AIMD runs using the gas phase linker or the full periodic MOF material. During the periodic MD simulations, the organic linkers experience interactions with the other linkers or inorganic units in the unit cell. The resulting dynamically obtained average excitation energy of the extracted organic linker includes both the induced effects 
Table 1 Vertical excitation energies of the organic linkers computed using TD-DFT (B3LYP/6-311+G(d,p)) on geometries obtained using a static calculation ('static') or from an MD run ('dynamic'). Additionally, the shift between the dynamic and static values are included for further reference. All values are in $\mathrm{nm}$

\begin{tabular}{|c|c|c|c|c|c|c|}
\hline & \multicolumn{3}{|c|}{ Gas phase } & \multicolumn{3}{|c|}{ Periodic } \\
\hline & Static & Dynamic & Shift & Static & Dynamic & Shift \\
\hline UiO-66 & 285 & 289 & 4 & 288 & 313 & 25 \\
\hline UiO-66-OH & 339 & 343 & 4 & 345 & 364 & 19 \\
\hline UiO-66- $\mathrm{NH}_{2}$ & 389 & 387 & -2 & 385 & 385 & 0 \\
\hline UiO-66-2,5OH & 411 & 412 & 1 & 417 & 446 & 29 \\
\hline UiO-66-2,5 $\mathrm{NH}_{2}$ & 528 & 502 & -26 & 467 & 490 & 23 \\
\hline UiO-66-oxal $^{\mathrm{a}}$ & - & - & & 341 & 342 & 1 \\
\hline $\mathrm{UiO}-66-\mathrm{CC}^{\mathrm{a}}$ & - & - & & 341 & 348 & 7 \\
\hline UiO-67 & 314 & 309 & -5 & 318 & 320 & 2 \\
\hline UiO- $67-2,5^{\prime} \mathrm{NH}_{2}$ & 387 & 376 & -11 & 383 & 385 & 2 \\
\hline
\end{tabular}

a The gas phase MD simulations have not been computed given the hypothetical nature of these organic linkers of the periodic lattice and of the harmonic and anharmonic nuclear motions.

Inspection of Table 1 reveals that when comparing the excitation energies based on the different geometries, no general trend can be derived for this set of isoreticular MOFs. For instance, the dynamic averages are not necessarily larger compared to the static values. Nevertheless, the following observations can be made.

First, the static excitation energies of the linkers obtained from a direct gas phase optimization ('gas phase/static') or extracted from the optimized periodic MOF ('periodic/ static') differ very minorly. This indicates that during geometry optimization of the full $\mathrm{UiO}$ frameworks, the linkers are not substantially altered as compared to an optimization of the isolated linker. Only in case of the BDC$2,5 \mathrm{NH}_{2}$ linker a large difference is found, which can be attributed to the different orientation of the hydrogen atoms of the $\mathrm{NH}_{2}$ substituents. In case of the static optimization, the hydrogens are located in the plane of the aromatic ring, whereas this is not the case for the linker extracted from the optimized UiO-66-2,5 $\mathrm{NH}_{2}$ material.

Second, the dynamic averages obtained from the AIMD runs show larger differences when comparing the gas phase ('gas phase/dynamic') and extracted ('periodic/dynamic') linkers. The difference ranges between 2 and $34 \mathrm{~nm}$. This already indicates that, as expected, the geometrical structure of the linkers is influenced by the periodic MOF framework during an MD simulation.

A final important observation from Table 1 is that the static and dynamic excitation energies can differ substantially, in particular for the periodic data. For example, for the UiO-66-OH and UiO-66-2,5OH materials, the dynamic AIMD values are 19 and $29 \mathrm{~nm}$ larger compared to their static results, respectively. These shifts are due to changes in the geometrical structures induced during the dynamic simulations and will be examined in detail in Sects. 4.2 and 4.3 by linking the excitation energies to the vibrational features of the material.

In addition to the calculated excitation energies, also the intensities of the electronic transitions can be taken into account. The dynamic averages are systematically lower compared to the static intensities, as shown in Fig. 4. From Fig. 4, the bathochromic shift upon using a electron-donating substituent is also clearly observable, in line with previous findings on these UiO-type materials [23].

The origin of the vertical transitions can be investigated based on the involved orbitals of the linkers. We previously reported that for the UiO-66-variants, the transition is mainly due to a HOMO to LUMO excitation, in which the contribution of the functional group almost vanishes in the LUMO orbital [23]. A similar observation can be made in the case of the longer BPDC-2,5 ${ }^{\prime} \mathrm{NH}_{2}$ linker, where the contribution of the electron-donating substituents reduces from $33 \%$ in the HOMO to $1 \%$ in the LUMO (see Fig. 5).

The optimized gas phase BPDC linkers (shown in Fig. 5) are nonplanar in the ground state, exhibiting a dihedral angle of 30.1 and $52.2^{\circ}$ of the two aromatic rings for BPDC and BPDC-2,5 $\mathrm{NH}_{2}$, respectively. The additional $\mathrm{NH}_{2}$ substituents increase the deviation from planarity. Some of the presenting authors previously investigated the influence of the torsional angle on the excitation energy in the case of a bipyridine-based linker [62]. For biphenyl, it is well described in the literature that the dihedral angle between the phenyl linkers is nonzero [36]. Importantly, this deviation from planarity in case of the BPDC linkers hampers the conjugation and the calculated excitation energies are hence not necessarily larger compared to their BDC counterparts (as indeed seen in Table 1). Moreover, when anchored in the UiO-67 (Fig. 6a) this torsional dihedral will be influenced. Notably, also the dihedral angle between the 

excitation spectra calculated on the linkers extracted from the periodic frameworks using static (full curves) and dynamic (dotted curves) methodologies. The intensities are expressed in $\mathrm{L} / \mathrm{mol} \mathrm{cm}$. The wavelength values of the peaks are indicated in Table 1: static ('periodic/ static') and dynamic ('periodic/ dynamic')
Fig. 4 Comparison of the
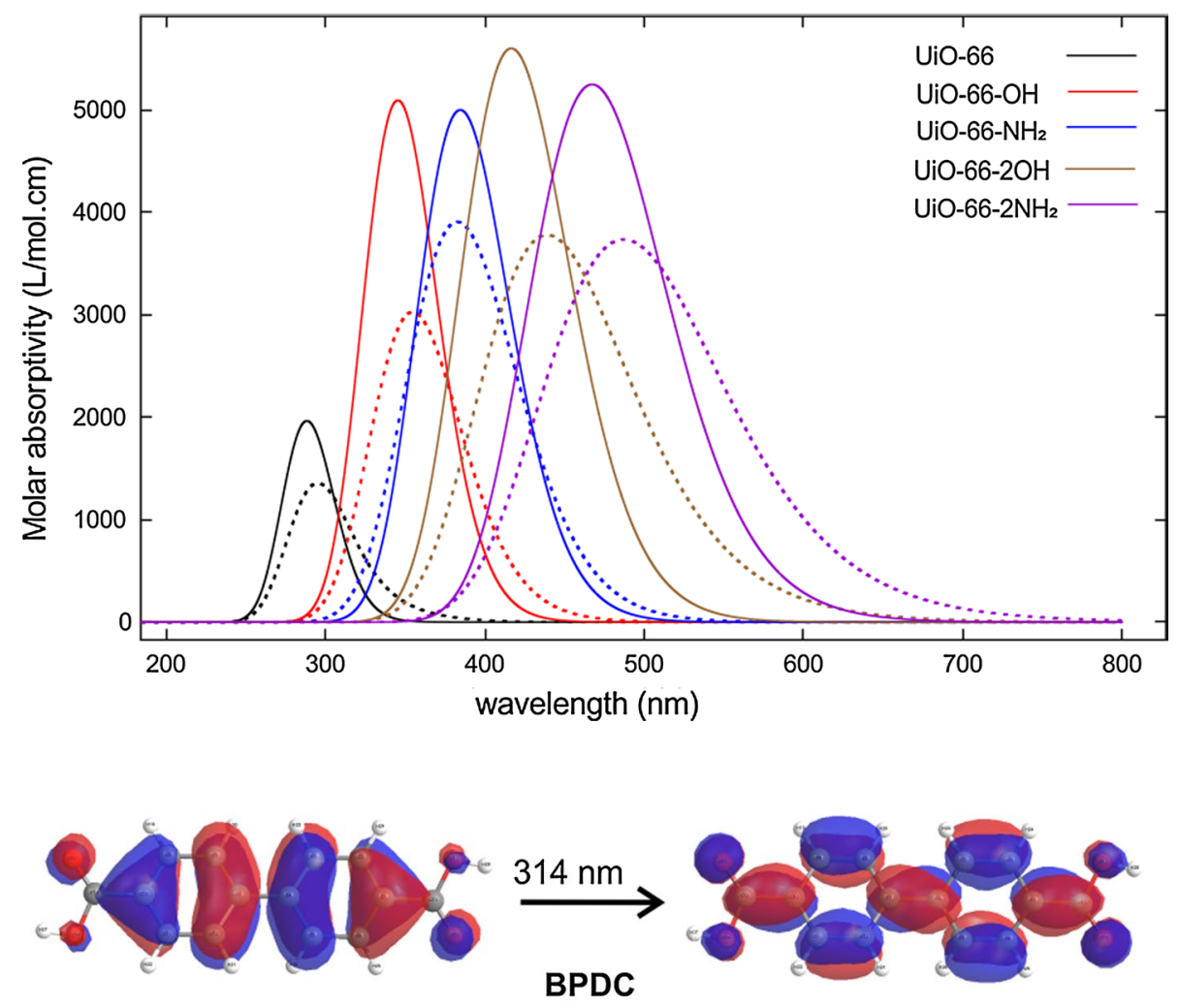

Fig. 5 Representation of the HOMO and LUMO orbitals involved in the first (vertical) electronic excitation of the BPDC and BPDC- $2,5^{\prime} \mathrm{NH}_{2}$ linker. The isosurfaces are shown for the gas phase optimized geometry of the linker

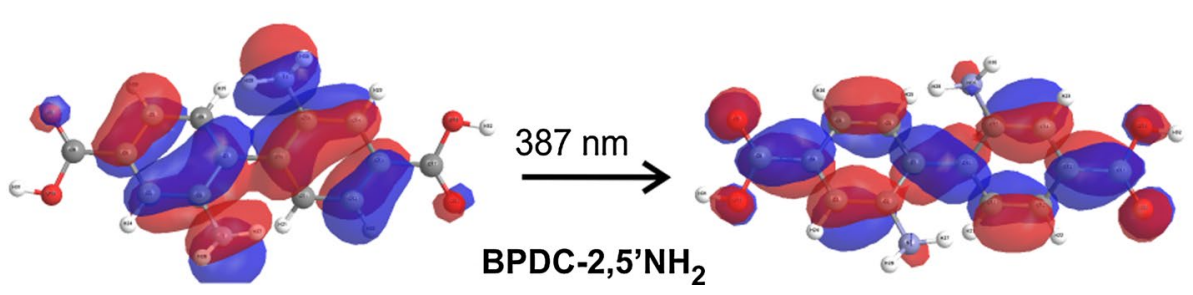

phenylic groups and the carboxylic parts of the linker influences the conjugation of the system. Both dihedral angles $(\alpha$ and $\beta$ ) are indicated in Fig. 6b. During the MD simulations, the spread in the values for these dihedral angles becomes substantially larger due to the increased temperature. Their distribution for the UiO-67 and UiO-67-2,5' $\mathrm{NH}_{2}$ materials, obtained during the gas phase and periodic MD simulations are displayed in Fig. 6c. It is observed that for UiO-67 the angles $\alpha$ and $\beta$ behave similarly when comparing the gas phase (full curves) and periodic (dotted curves) MD simulations. The dihedrals vary around an average value with ranges of about 60 and $80^{\circ}$ for $\alpha$ and $\beta$, respectively. For the functionalized linker, the dihedral angles are significantly larger due to the sterical hinder caused by the amine groups. For the gas phase BPDC- $\mathrm{NH}_{2}$ linker, the barrier between the two symmetry-related structures can be overcome during the MD simulations at $300 \mathrm{~K}$, resulting in the observed peaks at 70 and $110^{\circ}$ (which essentially represent the same structure). For the gas phase MD simulations, the average of $\beta$ is approximately zero, similar to the BDC linkers (see Fig. S4 in the Supplementary Material).
When placed in the framework, however, the carboxylic groups are bound to the inorganic clusters and thus much more rigid. Therefore, the dihedral angle between carboxyl group and the phenyl linkers is nonzero in the periodic simulations. This effect is even more pronounced for the $\mathrm{NH}_{2}$ -functionalized UiO-67 material, due to the larger torsional angle $\alpha$ between the phenyl rings.

\subsection{Vibrational analysis}

The MD trajectories are used to calculate VPS spectra, providing insight in the vibrational modes of the UiOs. A detailed analysis of the vibrational motions of all investigated structures is outside the scope of this paper, and therefore, only the main features are described.

Figure $7 \mathrm{a}$ depicts the VPS of the prototype UiO-66 material computed using the periodic MD trajectory. To gain more insight in the origin of the calculated VPS, partial VPS spectra, separating, e.g., the contributions from the linker and the inorganic $\mathrm{Zr}-\mathrm{O}$ unit, are also shown. The clear peak at $3731 \mathrm{~cm}^{-1}$ corresponds with the $\mathrm{OH}$ 
Fig. 6 a Periodic structure of the UiO-67 porous framework (using the same color code as Fig. 1). b Detailed view on the BPDC linker anchored at the inorganic $\mathrm{Zr}-\mathrm{O}$ units. Dihedrals $\alpha$ and $\beta$ represent the torsional angle between the two aromatic rings and the dihedral angle between a phenylic and carboxylic part, respectively. c Relative occurrence of the dihedral angles $\alpha$ and $\beta$ as calculated form the MD simulations using the fully periodic $\mathrm{UiO}-67$ (in blue) and $\mathrm{UiO}-67-\mathrm{NH}_{2}$ (in green) frameworks. Full curves obtained from gas phase MD and dotted curves from periodic MD simulations (a)
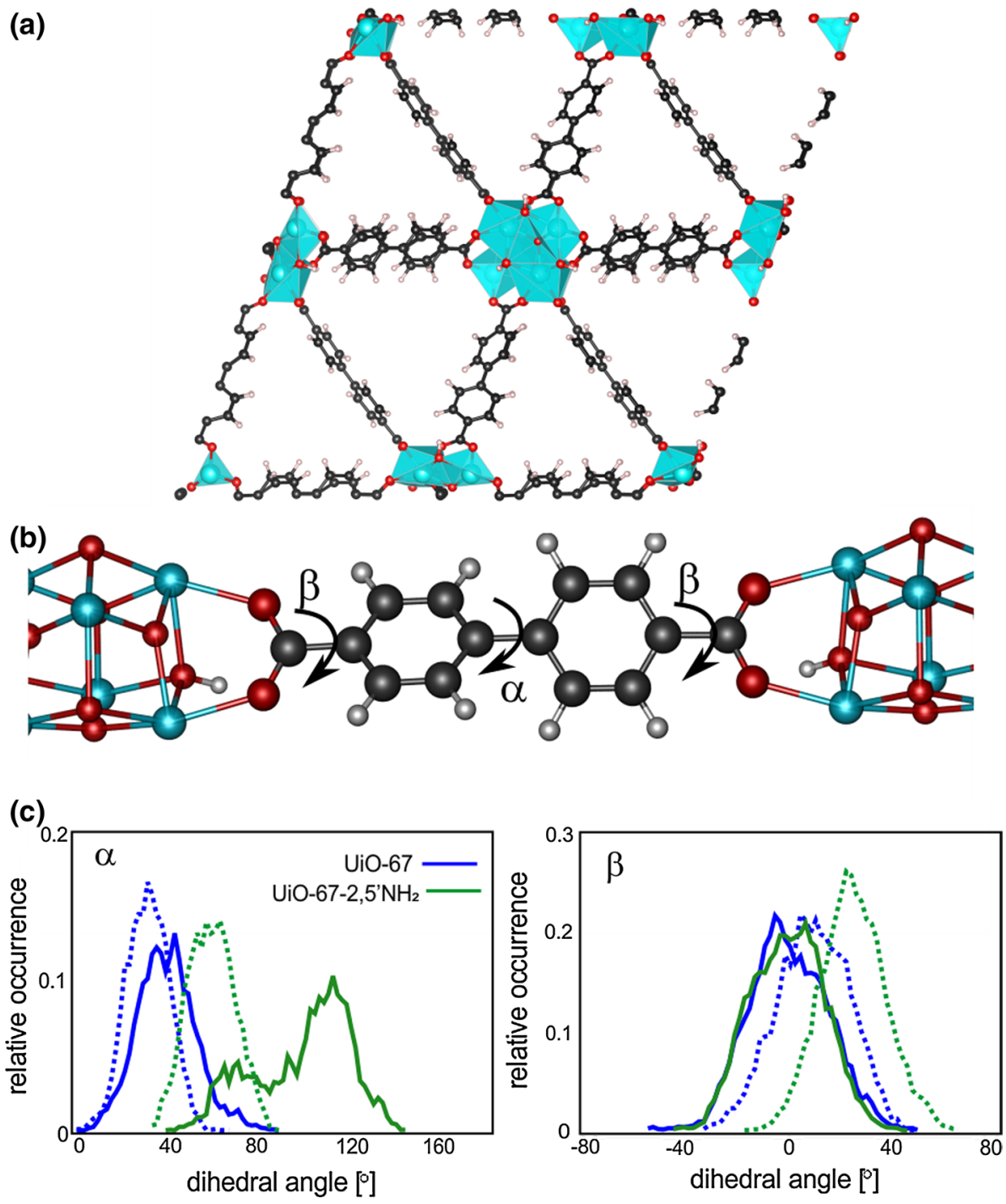

stretching frequency in the $\mathrm{Zr}_{6} \mathrm{O}_{4}(\mathrm{OH})_{4}$ units, denoted as the $\mu_{3}-\mathrm{OH}$. This value can be related with the experimental value of $3676 \mathrm{~cm}^{-1}$ reported by Lamberti et al., who combined static periodic B3LYP calculations with experimental data to report vibrational data of the UiO-66 [19] and UiO-67 [39] materials. The $\mathrm{CH}$ stretching frequencies of the aromatic BDC linker are located between 3100 and $3200 \mathrm{~cm}^{-1}$, but these are not expected to be of further importance in our discussion relating the vibrational motions to the changes in the excitation energy. The skeletal vibrations are situated at lower frequencies, i.e., between 400 and $1600 \mathrm{~cm}^{-1}$, and clearly these are dominated by the linker contributions. These contributions can mainly be assigned to various kinds of ring vibrations, including out-of-plane vibrations (i.e., at $400 \mathrm{~cm}^{-1}$ ), distortions and $\mathrm{C}-\mathrm{C}$ stretches. The partial VPS show that the vibrations of the $\mathrm{Zr}_{6}$-octahedron units are found between $400-470$ and $650-850 \mathrm{~cm}^{-1}$.
The VPS of UiO-66 also reveals three main peaks at very low wave numbers, i.e., at 38,50 and $60 \mathrm{~cm}^{-1}$ (see Fig. 7b). These so-called soft modes are associated with the carboxylate torsion and other global modes of the organic linkers, as made clear via PCA and visualized in Fig. 7c. These modes have an important influence on the calculated absorption properties, as shown hereafter in Sect. 4.3. The VPS provide an interesting way to compute these modes.

The selected test set allows to investigate the influence of both linker substitution and usage of extended linkers. Changes in the linker functionalization are most clearly observed in the stretching region above $3000 \mathrm{~cm}^{-1}$ as shown in Fig. 8a. Importantly, the calculated $v(\mathrm{O}-\mathrm{H})$ stretching frequencies of the $\mathrm{OH}$-substituted UiO-66s are found between 3250 and $3450 \mathrm{~cm}^{-1}$, indicative of mild hydrogen bonding in these structures. This is also revealed when comparing the VPS calculated from the MD of the gas phase linker with the VPS calculated for the linker 
Fig. 7 a Power spectra computed using MD of the fully periodic UiO-66 framework (black). Partial spectra using only the information from atoms of the inorganic (cyan), organic (gray) and carboxylic (orange) units are also shown. b Detail of the VPS of the periodic UiO-66: The region below $80 \mathrm{~cm}^{-1}$, indicative of the soft modes, is shown. c Representation of the characteristic soft modes, corresponding with global motions of the aromatic linker

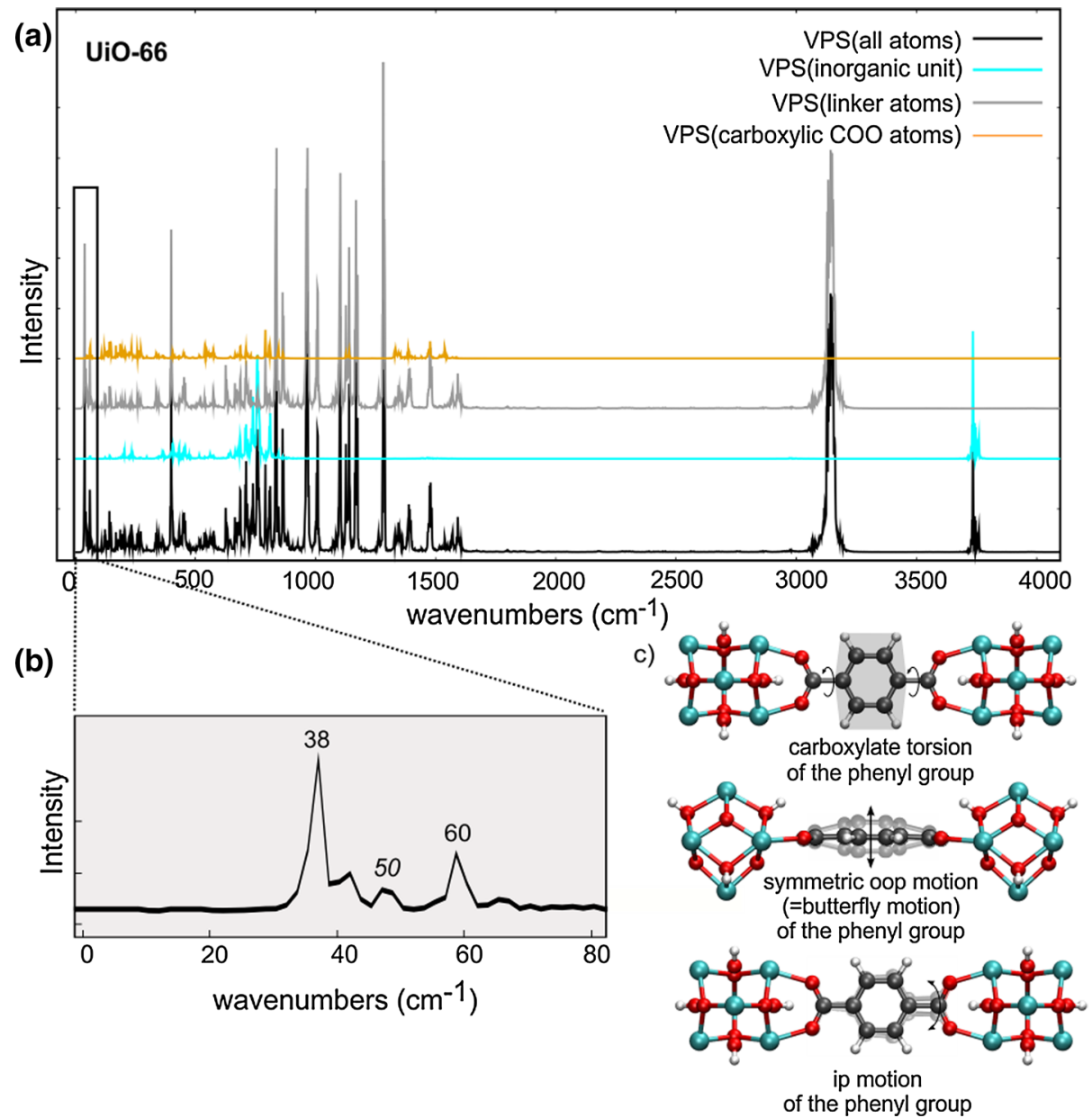

extracted from the MD of the periodic UiO framework, as illustrated in Fig. 8b for UiO-66-2,5OH. For the gas phase $\mathrm{MD}$, a substantial shift of the $\mathrm{OH}$ frequency is found compared to the periodic simulations. This is due to a stronger hydrogen bond with the carboxylic oxygens, reducing the strength of the observed $\mathrm{OH}$ bond more in the case of gas phase simulations. Inspection of the relevant geometrical parameters does, however, not result in changes between the values obtained from a static optimization and from dynamic averages (see Table S3 of the Supplementary Material).

Use of the longer BPDC linkers results in VPS which are very similar to the spectra of the UiO-66 materials (see Fig. S6 of the Supplementary Material). Detailed investigation shows that the VPS of the UiO-67 framework show an extra peak at $1188 \mathrm{~cm}^{-1}$, which must be attributed to a collective mode of the BPDC linker, in excellent agreement with the theoretical value of $1180 \mathrm{~cm}^{-1}$ reported by Chavan et al. [39]. In analogy with the VPS of UiO-66, the VPS of UiO-67 shows dominant peaks at low frequencies. Due to the occurrence of the biphenylic unit, a variety of low-frequency modes-including a butterfly mode, torsions over $\alpha$ and $\beta$-is present in the range below $70 \mathrm{~cm}^{-1}$. The VPS of the two models systems, UiO-66-oxal and UiO-66-CC (see Fig. S7 of the Supplementary Material), still show a lot of vibrational peaks in the skeletal region below $900 \mathrm{~cm}^{-1}$. Notably, the first peak of UiO-66-located around $38 \mathrm{~cm}^{-1}$ and corresponding with a global torsion of the organic aromatic linker-is now absent. A peak around $68 \mathrm{~cm}^{-1}$ represents a global translation of the central $\mathrm{CC}$ out of the carboxylic plane, motions which are indeed still present in both model systems. The $\mathrm{C} \equiv \mathrm{C}$ stretching mode in case of $\mathrm{UiO}-66-\mathrm{CC}$ is found at $2255 \mathrm{~cm}^{-1}$.

\subsection{VPS versus $\epsilon$ PS analysis}

To gain more insight into the motions contributing to the shift between the static and dynamic excitation energies, the VPS must be compared directly with the $\epsilon$ PS (see Sect. 2). The $\epsilon \mathrm{PS}$ reveal the modes that are characteristic for changes in the computed excitation energy. The $\epsilon \mathrm{PS}$ of the investigated 9 UiOs are depicted in the Supplementary Material (Fig. S8). All power spectra based on the periodic MD show dominant features at low-frequency modes, i.e., below $100 \mathrm{~cm}^{-1}$. These modes correspond with the global motions of the aromatic/aliphatic linkers, as visualized 
Fig. 8 Computed VPS using MD on the periodic UiOs. a Detail of the stretching region of the VPS of all functionalized UiO-66 frameworks. b Detailed $\mathrm{O}-\mathrm{H}$ stretching region of the UiO-66-2,5OH MOF and BDC2,50H linker
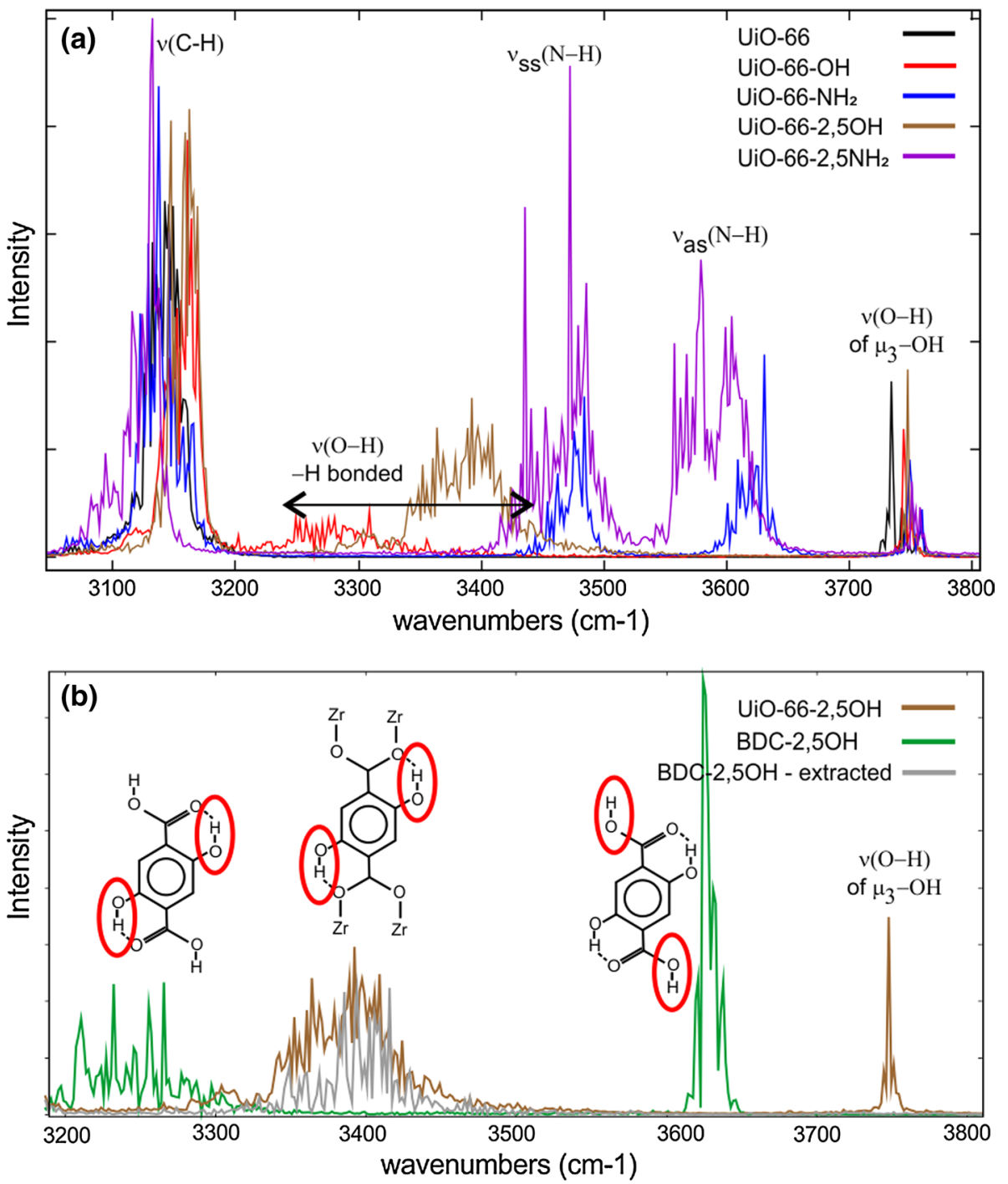

in Fig. 7c for the aromatic BDC unit. In the model case of UiO-66-oxal, such modes are less present, which has clearly its influence of the $\epsilon \mathrm{PS}$, yielding more pronounced peaks at higher frequencies. Other contributions to the $\epsilon$ PS resulting from these organic moieties are the vibrations located between 1400 and $1600 \mathrm{~cm}^{-1}$, representing, e.g., a variety of ring distortions in case of the aromatic units. Figure 9 shows the $\epsilon$ PS and VPS in case of (a) UiO-66, (b) UiO-66-CC, (c) UiO-66-2,50H and (d) UiO-67, in line with the conceptual Fig. 3. Detailed inspection reveals that although a correlation between the $\epsilon$ PS and VPS can overall be found, the complexity of the VPS hampers straightforward unraveling which vibrational modes are represented and a PCA is performed. In what follows, the main observations are summarized for the four case studies shown in Fig. 9.

In case of the original UiO-66 material (Fig. 9a), the $\epsilon \mathrm{PS}$ look rather simple, featuring a main peak around $50 \mathrm{~cm}^{-1}$, a smaller one at $38 \mathrm{~cm}^{-1}$ and some minor contributions between 60 and $125 \mathrm{~cm}^{-1}$ and around 1300-1600 $\mathrm{cm}^{-1}$. Close comparison between the $\epsilon \mathrm{PS}$ and the VPS reveals that all the peaks above $1300 \mathrm{~cm}^{-1}$ coincide in both power spectra. All these modes correspond with various in-plane ring vibrations. This indicates a linear dependency of the excitation property on changes in these coordinates. When averaging during the MD sampling, no net influence is observed on the average dynamic excitation energy compared to the static value. But, comparing the $\epsilon \mathrm{PS}$ with the $\epsilon \mathrm{PS} / 2$ (2 upper curves in Fig. 9a) reveals that several lowfrequency modes have both a linear and quadratic behavior since the spectra have coinciding peaks. Table 2 identifies the PCA coordinates for which the $Q_{i} \mathrm{PS}$ show high overlap with the scaled and unscaled $\epsilon \mathrm{PS} . Q_{2}$ and $Q_{3}$ are nearly degenerate with main peaks at 50 and $60 \mathrm{~cm}^{-1}$, with additional peaks for $Q_{3}$. Due to this degeneracy, these modes are not simple linear or quadratic modes. They are instead a mixture of a butterfly motion-quadratic - and an in-plane translation of the phenyl ring-linear motion-as shown in 
Fig. 9 Power spectra ${ }^{a}$ of selected UiO frameworks: a UiO-66, b • UiO-66-CC, c UiO-66-2,5OH and d UiO-67. Partial VPS from the periodic AIMD data, using only the linker atoms (black), $\epsilon \mathrm{PS}$ (dark gray) and $\epsilon \mathrm{PS}$ with scaling of the frequency axis by $1 / 2$ (light gray). A green tick indicates a correlation between the power spectra, whereas a red cross indicates no correlation is found. ${ }^{a}$ The TD-DFT simulations are solely performed on the linkers (either obtained from the gas phase MD or extracted from the periodic MD), and hence, the partial VPS using the linker atoms are employed (see Fig. 7a for UiO66). For the simulation of the VPS, the hydrogen atoms are neglected since their contribution to the VPS is dominant, while their influence on the $\pi$-system is very small

Fig. 7c. Although this degeneracy complicates the analysis, due to the presence of the peaks in both the scaled and nonscaled $\epsilon \mathrm{PS}$, we can safely conclude that the butterfly motion is quadratic and causes the absorption shift as seen in Table 1. Other modes, such as the global linker torsion $\left(Q_{1}\right)$ and in-plane phenyl rotation $\left(Q_{10}\right)$, have inherently both a quadratic and linear dependency, due to the saturation of the linker with hydrogen atoms in case of the linkers extracted from the periodic UiOs. In case of the MD simulations involving the gas phase BDC linker, the $\epsilon$ PS shows less activity in the low-frequency range. This indicates that no quadratic motions are found and, indeed, the static and dynamic excitation energy in case of the gas phase MD simulation is very similar (see Table 1).

The $\epsilon \mathrm{PS}$ of the model framework UiO-66-CC show seven main peaks (see Fig. 9b). The highest peak is located at low wave numbers (i.e., at $65 \mathrm{~cm}^{-1}$ ). Another peak in the $\epsilon \mathrm{PS}$ are located at $2255 \mathrm{~cm}^{-1}$, indicating that the $\mathrm{C} \equiv$ $\mathrm{C}$ stretch of the model linker also impacts changes in the excitation energy. Again, for all peaks an overlap between the $\epsilon$ PS and VPS is found, demonstrating a linear correlation along these modes, and thus, a small overall shift is obtained between the dynamic average and static value of the excitation energy (the obtained shift indeed only equals $7 \mathrm{~nm}$, see Table 1).

Due to the complexity of the VPS of UiO-66-2,5OH and UiO-67 (Fig. 9c, d), a PCA is performed. For these materials, Tables S4-S7 of the Supplementary Material list all peaks observed in the $\epsilon \mathrm{PS}$ spectra with the corresponding modes obtained from PCA. In addition to the peak positions, a description of the vibrational motion is given. For the UiO-66-2,50H framework, the excitation energy computed using a static or dynamic approach differs significantly in the case of the AIMD of the fully periodic MOF: a bathochromic shift of $29 \mathrm{~nm}$ is obtained (see Table 1). As this shift is not observed in case of the AIMD of the gas phase BDC-2,5OH linker, an important effect of the periodic framework is suggested. In case of the periodic AIMD data, the PCA reveals the occurrence of a quadratic
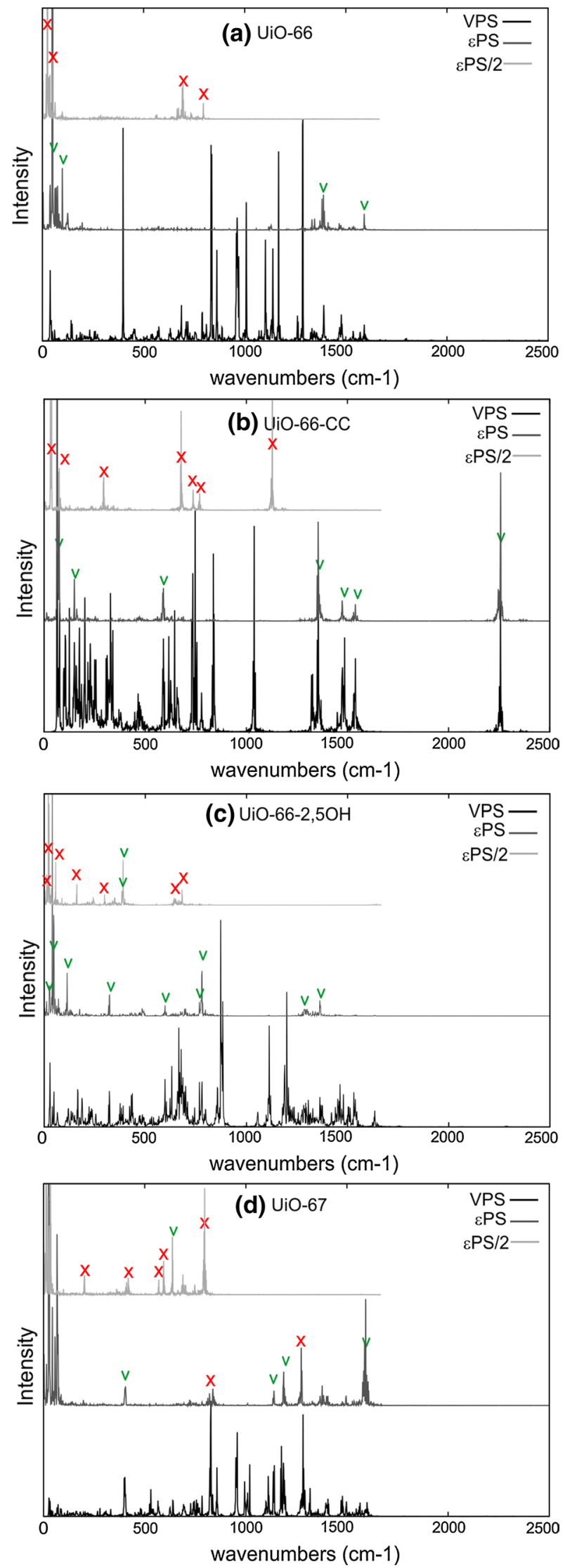
Table 2 Assignment table of linear and quadratic PCA modes $\left(Q_{i}\right)$ for the UiO-66 material, using the periodic AIMD data
Fig. 10 Comparison of the $\epsilon \mathrm{PS}$ with scaling of the frequency axis by $1 / 2$ and the $Q_{11} \mathrm{PS}$ obtained from the PCA in case of UiO-66-2,5OH. The corresponding motion is visualized (hydrogen atoms are omitted for clarity). The periodic AIMD data were used

\begin{tabular}{lcccccccccc}
\hline & $\epsilon \mathrm{PS}$ & $Q_{1}$ & $Q_{2}$ & $Q_{3}$ & $Q_{10}$ & $\epsilon \mathrm{PS} / 2$ & $Q_{1}$ & $Q_{2}$ & $Q_{3}$ & $Q_{10}$ \\
\hline 1 & 38 & 38 & & 38 & & 19 & & & & \\
2 & 50 & & 50 & 50 & & 25 & & & & \\
3 & 62 & & 60 & 60 & 62 & 31 & & & & \\
4 & 68 & & & 68 & & 34 & & & & \\
5 & 75 & & & & & 37.5 & 38 & & 38 & \\
6 & 98 & & & & 49 & & 50 & 50 & \\
7 & 125 & & & & & 62.5 & & 60 & 60 & 62 \\
\hline
\end{tabular}

The characteristic peaks of the $\epsilon$ PS are given for the linearly (unscaled) and quadratically (scaled with $1 / 2$ ) active modes. The peaks of the $Q_{i} \mathrm{PS}$ are also listed. All data are given in $\mathrm{cm}^{-1}$

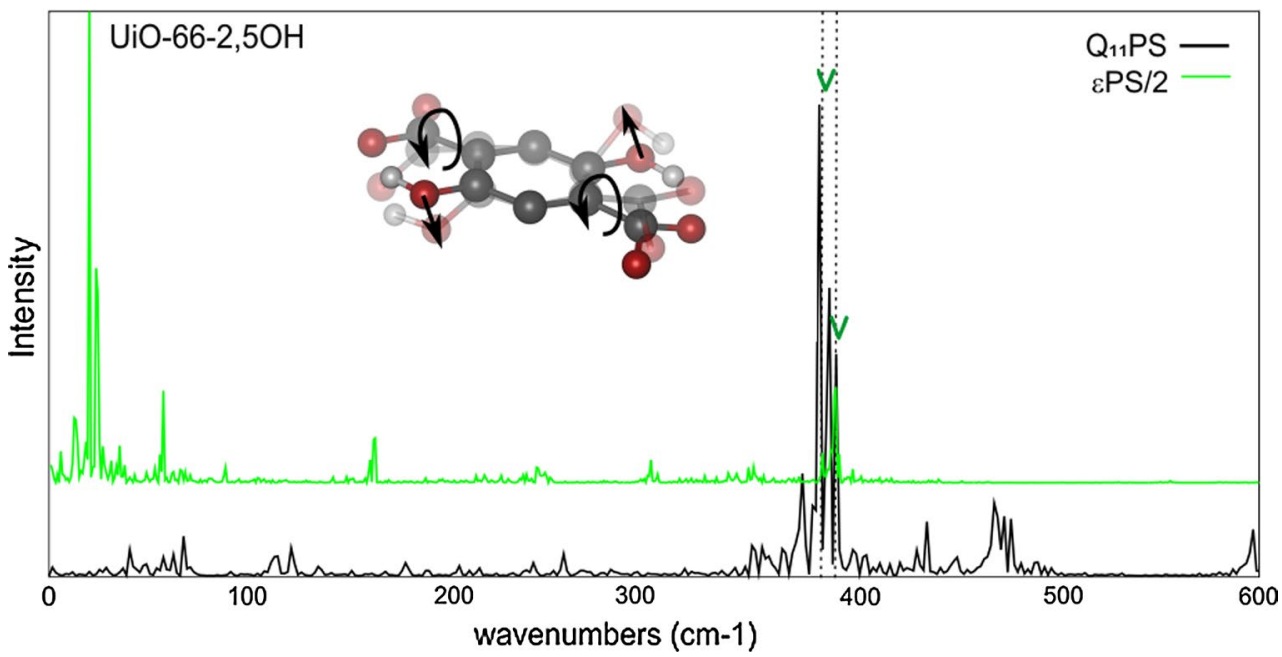

coordinate $Q_{11}$, of which the corresponding power spectrum $\left(Q_{11} \mathrm{PS}\right)$ exhibits peaks at 381 and $390 \mathrm{~cm}^{-1}$ (see Table S5 in the Supplementary Material). These peaks are also found in the $\epsilon \mathrm{PS}$ with the frequency axis scaled with 1/2 (see Fig. 10). These peaks are due to a complicated motion, including the asymmetric out-of-plane motion of carbon and oxygen atoms of the BDC unit and $\mathrm{OH}$ substituents as shown in Fig. 10. This vibrational mode dominates the shift between the averaged dynamic and static excitation energy. For the gas phase AIMD data, all coordinates exhibit a linear behavior (see Table S4), rationalizing that the dynamic average almost equals the value obtained from the static optimization.

The UiO-67 framework offers another interesting case study. Clearly, motions affecting the central $\mathrm{C}-\mathrm{C}$ bond disturb the $\pi$-system of the conjugated aromatic linker substantially. These modes are expected to have a significant influence on the resulting excitation energy. The analysis of the $\epsilon$ PS involving the gas phase AIMD is already rather complicated due to the large number of peaks (see Table S6), confirming that many vibrations impact the excitation energy. The biphenylic unit results in a variety of motions that can occur, and importantly, many of them exhibit a quadratic behavior. Notably, the butterfly motion, torsion of the phenylic rings and ring vibrations (e.g., the in-plane ring vibration at $631 \mathrm{~cm}^{-1}$ ) are dominant modes. Inclusion of the framework in the AIMD simulations leads to a rather similar picture, although fewer quadratic modes are obtained. The $\epsilon \mathrm{PS}$ of the linkers extracted from the UiO67 framework show 4 main contributions in the very lowfrequency range, i.e., below $70 \mathrm{~cm}^{-1}$, but only one of these peaks is now quadratic (see Table S7). Using PCA, it can be concluded that this peak represents the global rotation of the biphenyl linker compared to the rest of the UiO-67 framework (i.e., rotating over the dihedral angle $\beta$, see Fig. 6b). A second quadratic peak is found at $637 \mathrm{~cm}^{-1}$ and corresponds with an in-plane vibration of the aromatic rings. In these cases, the various quadratic contributions might compensate each other, and hence, the observed shift between the static and dynamic excitation energy is found to be relatively small (see Table 1). The case studies above indicate that the analysis based on power spectra can identify the vibrational modes that impact the calculated excitation energy. 


\section{Conclusions}

In this paper, theoretical simulations have been employed to assess which vibrational modes impact absorption properties of UiO-type metal-organic frameworks. The excitation energies have been calculated using TD-DFT on the organic linkers. Nine UiO-based frameworks have been tested, varying in linker substituents-considering electron-donating $\mathrm{OH}$ and $\mathrm{NH}_{2}$ substituents-and linker length. In addition to the UiO-66 and UiO-67 materials and substituted derivatives, two model systems exhibiting a simplified linker have been considered. The impact of nuclear motions on the excitation properties have been accounted for by sampling the potential ground-state surface using ab initio molecular dynamics (AIMD) simulations. Subsequently, averaged dynamic absorption properties have been calculated and compared with absorption data from an optimized geometry. The sampling has been done for both the gas phase organic linker and the fully periodic UiO framework. Given the distinct simulations involving various sets of generated geometries, the analysis allows addressing the influence of the periodic framework, temperature and harmonic and anharmonic nuclear motions.

The absorption spectra of the mono- and disubstituted UiO-66 MOFs show a clear bathochromic shift upon substitution. In case of UiO-67, $\mathrm{NH}_{2}$ substitution results in a similar modulation and also indicates that the contribution of the substituent to the LUMO nearly vanishes in the HOMO to LUMO excitation. Velocity power spectra, calculated from the MD trajectories, provide vibrational information of the UiO materials. For the UiO-66-OH and UiO-66-2,5OH frameworks, the VPS provide insight into the hydrogen bonding in these materials and an influence of the framework is reported. The soft modes at low frequencies can be calculated for all UiOs with accuracy, providing characteristic values below $70 \mathrm{~cm}^{-1}$ for the global linker motions, such as ring torsions and butterfly motions.

The VPS are directly compared with the power spectra of the excitation energy $\epsilon \mathrm{PS}$ to identify the dominant vibrational motion(s) responsible for changes in the spectroscopic property. Inspection of the $\epsilon \mathrm{PS}$ reveals that for all periodic UiO frameworks, the low-frequency modes impact the calculated excitation energy. In case of the periodic UiO-66 material, the shift between the static and dynamic excitation energy mainly originates from the global butterfly motion of the organic linker. For the periodic UiO-66$2,5 \mathrm{OH}$ material, a more complicated motion, including outof-plane movements of atoms of both the aromatic unit and the substituents, is identified. These shifts are not observed when the dynamics solely involve the gas phase organic linker, clearly pointing out the effect of the periodic $\mathrm{UiO}$ framework. For the UiO-67 material, the torsion between the phenylic rings also impacts the excitation energy. The computational tool based on power spectra allows to determine the vibrational fingerprint of excitation properties of nanoporous materials featuring global modes such as $\mathrm{UiO}$ frameworks. The resulting insights based on a combination of MD and TD-DFT simulations add to the complete understanding of the light absorption properties of these complex materials.

\section{Supplementary Material}

Computational details of the ab initio periodic simulations and optimized cell parameters. Details on the termination procedure of the extracted linkers. Absorption intensities. Structural parameters, including bond lengths, angles and dihedrals of selected UiOs comparing the static and dynamic simulations. VPS comparing UiO-66 and UiO-67 and UiO-66-CC and UiO-66-oxal. $\epsilon$ PS of all 9 UiOs. Comparison of VPS and $\epsilon$ PS of UiO-66, UiO-66-2,50H and UiO-67.

Acknowledgments The Fund for Scientific Research-Flanders (FWO), the Research Board of Ghent University (BOF, UGent GOA Grant 01G00710) and BELSPO in the frame of IAP/7/05 for financial support. V. Van Speybroeck acknowledges funding from the European Research Council under the European Community's Seventh Framework Programme (FP7(2007-2013) Grant Agreement No. 240483), and from the European Union's Horizon 2020 research and innovation program (consolidator ERC Grant Agreement No. 647755-DYNPOR (2015-2020)). Computational resources and services were provided by the Stevin Supercomputer Infrastructure of Ghent University and by the Flemish Supercomputer Center (VSC), funded by the Hercules Foundation and the Flemish Government-Department EWI. G.S. thanks the Spanish government for the provision of Severo Ochoa project (SEV 2012-0267).

\section{References}

1. Yaghi OM, O'Keeffe M, Ockwig NW, Chae HK, Eddauoudi M, Kim J (2003) Nature 423:705

2. Rowsell JLC, Yaghi OM (2004) Microporous Mesoporous Mater 73:3

3. Ferey G (2008) Chem Soc Rev 37:191

4. Kitagawa S, Kitaura R, Noro S (2004) Angew Chem Int Ed 43:2334

5. Furukawa H, Cordova KE, O'Keeffe M, Yaghi OM (2013) Science 341:974

6. Thematic issue on Metal-Organic Frameworks. Chem Rev 112(2):673-1268 (2012)

7. Corma A, García H, Llabrés i Xamena FX (2010) Chem Rev 110(8):4606

8. Liu D, Lu K, Poon C, Lin W (2013) Inorg Chem 53(4):1916

9. Wang J, Wang C, Lin W (2012) ACS Catal 2(12):2630

10. Nasalevich MA, van der Veen M, Kapteijn F, Gascon J (2014) Cryst Eng Commun 16(23):4919

11. Allendorf MD, Bauer CA, Bhakta RK, Houk RJT (2009) Chem Soc Rev 38(5): 1330 
12. Cavka JH, Jakobsen S, Olsbye U, Guillou N, Lamberti C, Bordiga S, Lillerud KP (2008) J Am Chem Soc 130(42):13850

13. Wiersum A, Estelle S, Yang Q, Moulin B, Guillerm V, Yahia M, Bourrelly S, Vimont A, Miller S, Vagner C, Daturi M, Clet G, Serre C, Maurin G, Llewellyn P (2011) Chem Asian J 6(12):3270

14. Vandichel M, Hajek J, Vermoortele F, Waroquier M, De Vos D, Van Speybroeck V (2014) Cryst Eng Comm 17(2):395

15. Zhao W, Zhang C, Yan Z, Bai L, Wang X, Huang H, Zhou Y, Xie Y, Li F, Li J (2014) J Chromatogr A 1370:121

16. Stassen I, Styles M, Assche T, Campagnol N, Fransaer J, Denayer J, Tan J, Falcaro P, De Vos D, Ameloot R (2015) Chem Mater 27(5):1801

17. Kim S, Lee Y, Hong S, Jang M, Ahn W (2015) Catal Today 245:54

18. Shen L, Wu W, Liang R, Lin R, Wu L (2013) Nanoscale 5:9374-9382

19. Valenzano L, Civalleri B, Chavan S, Bordiga S, Nilsen MH, Jakobsen S, Lillerud KP, Lamberti C (2011) Chem Mater 23(7): 1700

20. Gomes C, Silva, Luz I, Llabrés i Xamena FX, Corma A, García H (2010) Chem Eur J 16(36): 11133

21. Sun D, Fu Y, Liu W, Ye L, Wang D, Yang L, Fu X, Li Z (2013) Chem Eur J 19(42):14279

22. Hendrickx K, Vanpoucke DEP, Leus K, Lejaeghere K, Van Yperen-De Deyne A, Van Speybroeck V, Van Der Voort P, Hemelsoet K (2015) Inorg Chem 54(22):10701

23. Shen L, Liang R, Luo M, Jing F, Wu L (2014) Phys Chem Chem Phys 17(1):117

24. Flage-Larsen E, Røyset A, Cavka JH, Thorshaug K (2013) J Phys Chem C 117(40):20610

25. Musho T, Li J, Wu N (2014) Phys Chem Chem Phys 16(43):23646

26. Pavone M, Brancato G, Morelli G, Barone V (2006) ChemPhysChem 7:148

27. Barone V, Bloino J, Monti S, Pedone A, Prampolini G (2010) Phys Chem Chem Phys 12:10550

28. De Mitri N, Monti S, Prampolini G, Barone V (2013) J Chem Theory Comput 9:4507

29. Barone V, Biczysko M, Bloino J, Carta L, Pedone A (2014) Comput Theor Chem 1037:35

30. Pedone A, Prampolini G, Monti S, Barone V (2011) Chem Mater 23(22):5016

31. De Meyer T, Hemelsoet K, Van der Schueren L, Pauwels E, De Clerck K, Van Speybroeck V (2012) Chem Eur J 18(26):8120

32. Hemelsoet K, Qian Q, De Meyer T, De Wispelaere K, De Sterck B, Weckhuysen BM, Waroquier M, Van Speybroeck V (2013) Chem Eur J 19(49): 16595

33. Runge E, Gross EKU (1984) Phys Rev Lett 52(12):997

34. Marques M, Ullrich C, Nogueira F, Rubio A, Burke K, Gross E (2006) Time-Dependent Density Functional Theory. Springer, Berlin

35. Adamo C, Jacquemin D (2012) Chem Soc Rev 42(3):845

36. Van Yperen-De Deyne A, De Meyer T, Pauwel E, Ghysels A, De Clerck K, Waroquier M, Van Speybroeck V, Hemelsoet K (2014) J Chem Phys 140(3):134105

37. Lamberti C, Zecchina A, Groppo E, Bordiga S (2010) Chem Soc Rev 39:4951

38. Odoh SO, Cramer CJ, Truhlar DG, Gagliardi L (2015) Chem Rev 115:6051

39. Chavan S, Vitillo JG, Gianolio D, Zavorotynska O, Civalleri B, Jakobsen S, Nilsen MH, Valenzano L, Lamberti C, Lillerud KP, Bordiga S (2012) Phys Chem Chem Phys 14:1614
40. Van Speybroeck V, Hemelsoet K, Joos L, Waroquier M, Bell RG, Catlow CRA (2015) Chem Soc Rev. 44:7044. doi:10.1039/ c5cs00029g

41. Leus K, Concepcion P, Vandichel M, Meledina M, Grirrane A, Esquivel D, Turner S, Poelman D, Waroquier M, Van Speybroeck V, Van Tendeloo G, Garcia H, Van der Voort P (2015) RSC Adv 5:22334

42. Greathouse JA, Allendorf MD (2008) J Phys Chem C 112:5795

43. Verstraelen T, Van Houteghem M, Van Speybroeck V, Waroquier M (2008) J Chem Inf Model 48(12):2414

44. Van Houteghem M, Verstraelen T, Van Neck D, Kirschhock C, Martens JA, Waroquier M, Van Speybroeck V (2011) J Chem Theory Comput 7(4): 1045

45. Martinez M, Gaigeot MP, Borgis D, Vuilleumier R (2006) J Chem Phys 125:144106

46. Hutter J, Iannuzzi M, Schiffmann F, Joost V (2014) WIREs Comput Mol Sci 4(1): 15

47. Grimme S, Antony J, Ehrlich S, Krieg H (2010) J Chem Phys 132(15): 154104

48. Lippert G, Hutter J, Parrinello M (1997) Mol Phys 92(3):477

49. Hartwigsen C, Goedecker S, Hutter J (1998) Phys Rev B 58(7):3641

50. Goedecker S, Teter M, Hutter J (1996) Phys Rev B 54(3):1703

51. Bussi G, Donadio D, Parrinello M (2007) J Chem Phys 126(1):014101

52. Nosé S (1984) Mol Phys 52(2):255

53. Nosé S (1984) J Chem Phys 81(1):511

54. Yang Q, Guillerm V, Ragon F, Wiersum AD, Llewellyn PL, Zhong C, Devic T, Serre C, Maurin G (2012) Chem Commun 48:9831

55. Feyand M, Mugnaioli E, Vermoortele F, Bueken B, Dieterich JM, Reimer T, Kolb U, DeVos D, Stock N (2012) Angew Chem Int Ed 51(41): 10373

56. Frisch MJ, Trucks GW, Schlegel HB, Scuseria GE, Robb MA, Cheeseman JR, Scalmani G, Barone V, Mennucci B, Petersson GA, Nakatsuji H, Caricato M, Li X, Hratchian HP, Izmaylov AF, Bloino J, Zheng G, Sonnenberg JL, Hada M, Ehara M, Toyota K, Fukuda R, Hasegawa J, Ishida M, Nakajima T, Honda Y, Kitao O, Nakai H, Vreven T, Montgomery JA, Jr., Peralta JE, Ogliaro F, Bearpark M, Heyd JJ, Brothers E, Kudin KN, Staroverov VN, Kobayashi R, Normand J, Raghavachari K, Rendell A, Burant JC, Iyengar SS, Tomasi J, Cossi M, Rega N, Millam JM, Klene M, Knox JE, Cross JB, Bakken V, Adamo C, Jaramillo J, Gomperts R, Stratmann RE, Yazyev O, Austin AJ, Cammi R, Pomelli C, Ochterski JW, Martin RL, Morokuma K, Zakrzewski VG, Voth GA, Salvador P, Dannenberg JJ, Dapprich S, Daniels AD, Farkas, Foresman JB, Ortiz JV, Cioslowski J, Fox DJ (2009) Gaussian09 Revision D.01. Gaussian Inc. Wallingford, CT

57. Lee C, Yang W, Parr RG (1988) Phys Rev B 37(2):785

58. Becke AD (1993) J Chem Phys 98(7):5648

59. Krishnan R, Binkley J, Seeger R, Pople J (1980) J Chem Phys 72(1):650

60. Jacquemin D, Perpète E, Scuseria G, Ciofini I, Adamo C (2008) J Chem Theory Comput 4(1):123

61. Tomasi J, Mennucci B, Cammi R (2005) Chem Rev 105(8):2999

62. Liu YY, Decadt R, Bogaerts T, Hemelsoet K, Kaczmarek AM, Poelman D, Waroquier M, Van Speybroeck V, Van Deun R, Van Der Voort P (2013) J Phys Chem C 117:11302 